\title{
Biomimetic Metal-organic Framework Nanoparticles for Synergistic Combining of SDT-chemotherapy Induce Pyroptosis in Gastric Cancer
}

zhu yu ( $\square$ yuzhu676793@outlook.com )

Guangxi Medical University First Affiliated Hospital

\section{Wenlong Cao}

Guangxi Medical University First Affiliated Hospital

Chuangye Han

Guangxi Medical University First Affiliated Hospital

\section{Zhen Wang}

Guangxi Medical University First Affiliated Hospital

Yue Qiu

Guangxi Medical University First Affiliated Hospital

Jiancheng Wang

Guangxi Medical University First Affiliated Hospital

Junfu Wang

Guangxi Medical University First Affiliated Hospital

\section{Siwen Zhang}

Guangxi Medical University First Affiliated Hospital

\section{Senfeng Liu}

Guangxi Medical University First Affiliated Hospital

\section{Shutian Mo}

Guangxi Medical University First Affiliated Hospital Junqiang Chen

Guangxi Medical University First Affiliated Hospital

\section{Research Article}

Keywords: Nanoparticles, SDT-chemotherapy, Gastric Cancer, Pyroptosis

Posted Date: June 11th, 2021

DOI: https://doi.org/10.21203/rs.3.rs-594583/v1 
License: (c) (i) This work is licensed under a Creative Commons Attribution 4.0 International License. Read Full License

Version of Record: A version of this preprint was published at Frontiers in Bioengineering and Biotechnology on February 21st, 2022. See the published version at https://doi.org/10.3389/fbioe.2022.796820. 


\section{Abstract}

In recent years, sonodynamic therapy (SDT) has been widely developed for cancer research as a promising non-invasive therapeutic strategy. Here, we synthesized Zeolitic imidazole frameworks-8 (ZIF-

8) and utilized its properties to encapsulate hydrophobic Chlorin e6 (Ce6) and hydrophilic tirapazamine (TPZ) for a synergistic sonodynamic-chemotherapy, which was also accompanied by the modification of cytomembrane of gastric cancer (GC) cells. Thus, we enabled the biomimetic property to achieve targeted delivery. Ce6-mediated SDT, in combination with ultrasound irradiation, could target the release of reactive oxygen species (ROS) to aggravate further hypoxia, which activated TPZ. Combining these effects could induce the pyroptosis of GC cells. Both in vitro and in vivo experiments showed that the nanoparticle had good biocompatibility and anti-cancer function, which could provide a potential therapeutic method for cancer therapy.

\section{Introduction}

Every year over one million new cases of gastric cancer (GC) are diagnosed. Also, GC has already become the fifth most common cancer, globally [1]. The mortality in gastric cancer is high, and usually, it is diagnosed at an advanced stage, which makes it rank the fourth for mortality [1,2]. The main therapies for gastric cancer cover surgery, neoadjuvant chemotherapy, radiotherapy, chemoradiotherapy, immunotherapy and molecular-targeted therapy $[3,4]$. Despite these therapies, the 5-year overall survival rate remains low, with an approximate rate of $20 \%$ in most countries of the world [5]. Hence, a new potential therapeutic strategy for gastric cancer is yet to be explored.

Ce6 is a kind of organic sonosensitizer with low side effects, which can be better accumulated in tumors and also can be eliminated faster from the organisms. It a type of porphyrin that has properties of high sensitization with the capability to produce ROS [6]. Ce6 is reported to be activated by light/ultrasound and can inhibit the growth of tumor cells $[7,8]$. However, Ce6 has a property of hydrophobicity in the water environment, and since it gathers into a bigger crystal, it gets harder for it to function completely [9, 10]. Also, it can be cleared and degraded more rapidly in the long-term blood circulation [10]. Hence, there arises a question on how do we find a good way to deliver $\mathrm{Ce} 6$ for it to play an anti-tumor function.

Hypoxia or severe oxygen deprivation is considered as one evident challenge in the therapy of solid tumors because hypoxic regions are resistant to most anticancer drugs preventing the killing of tumor cells $[11,12]$. Tirapazamine (TPZ), a new class of bioreductive cytotoxic drugs, can be selectively toxic to hypoxia cells $[13,14]$. Due to the lack of oxygen within the cells, TPZ can be reduced to a toxic-free radical that can induce breaks in the cellular DNA [15]. TPZ has been put into use in phase II and III clinical studies [12]. and studies have proven that the combined use of TPZ and cisplatin could enhance the anti-tumor effect in malignant and non-small cell lung cancers [16]. Therefore, we should take the advantage of the hypoxic environment of a tumor to maximize the anti-tumor effects. 
ZIF-8 is one of the metal-organic frameworks (MOFs) synthesized using $\mathrm{Zn}^{2+}$ ion and 2methylimidazolate (2-MIL) [17]. It is known for its high surface area, tunable porosity, excellent chemical and thermal stability. It is also easy to synthesize it in water and alcoholic phases [18]. Especially, it has a $\mathrm{pH}$-responsive structure, which indicates its ease of degradation in an acidic environment along with the maintenance of steadiness in neutral conditions $[19,20]$. Due to these properties, ZIF-8 can be used as a framework to load drugs and target the release of drugs according to its response against the $\mathrm{pH}$ of the acidic tumor microenvironment [21]. Importantly, due to the strong electrostatic interaction of $\mathrm{Zn}^{2+}$ ion and Ce6, Ce6 can be easily loaded onto ZIF-8 by one-pot encapsulation [22]. Thus, ZIF-8 is usually applied in the research of cancer therapy.

SDT has advantages of greater safety, ease of operation, high precision, non-invasiveness, strong penetration into the deep tumor, and less side effects [23]. Especially, because of its strong penetration into deep tumors, it can be applied for deep tumor therapy, such as liver and pancreatic tumors. Hence, it has been widely used in cancer therapeutic research. SDT takes a combination of low-intensity ultrasound (US), $\mathrm{O}_{2}$, and sonosensitizers (all non-toxic) to generate a large quantity of ROS that can kill tumor cells [24-26]. A high concentration of ROS can kill tumor cells since it cannot be immediately cleared by the cells and its over-accumulation induces hypoxia and oxidative damage, leading to apoptosis, autophagy, or pyroptosis of cells $[6,27,28]$. However, a lot of $\mathrm{O}_{2}$ is required for SDT to play the anti-tumor function, and since most solid tumors have a hypoxic environment, it limits the anti-tumor efficacy. This problem can be solved by two methods. One is by utilizing the nanoparticles to carry $\mathrm{O}_{2}$ and help in killing tumor cells. The other one is by using nanoparticles to encapsulate chemotherapeutic drugs that are activated by hypoxia. After stimulating Ce6 by the US, ROS is produced, which consumes $\mathrm{O}_{2}$ in tumor cells, activating the hypoxia-activating drugs, which enhances the anti-tumor effect of SDT. Comparing with the traditional organic sonosensitizers, the nano-sonosensitizer, a type of sonosensitizer, has several advantages. Firstly, it can improve the properties of organic sonosensitizers. Secondly, it can prolong the circulation time of the drug in the blood, enabling more accumulation in the tumor site. Thirdly, it can strengthen the penetration retention, exerting a stronger therapeutic effect [29, 30]. Nanoparticle-assisted sonosensitizer is a type of nano-sonosensitizer, and with organic sonosensitizers loaded onto the nanoparticles, they are called nanoparticle-assisted sonosensitizers, which have the property of being released at the desired sites [23].

The study was aimed to synthesize a metal-organic framework namely ZIF-8, whose properties were utilized to encapsulate the hydrophobic Ce6 and TPZ. Simultaneously, we also wrapped it with homologous tumor cytomembrane as the outermost layer to enhance its targeting ability, i.e., its ability to be uptaken by the tumor cells. The whole nanoparticle was used as a nanoparticle-assisted sonosensitizer for SDT to kill the tumor cells. We hypothesized that when the nanoparticles entered the tumor microenvironment, the framework was degraded by acidic conditions, and Ce6 and TPZ were released. Irradiation by the US activated Ce6 and produced ROS to kill tumor cells. At the same time, the accumulation of ROS further aggravated hypoxia in the tumor microenvironment, which triggered TPZ and enhanced cell killing. Therefore, the whole anti-tumor effect was strengthened. 


\section{Results And Discussion}

\section{Characteristic of nanoparticles}

ZTC was prepared using zeolitic imidazolate framework 8 (ZIF-8) coloaded with ZTC and Ce6. The final nanoparticle called ZTC@M was prepared by extruding and wrapping cytomembrane fragments of AGS cell. TEM images showed that ZTC had the same structure as ZIF-8 (Fig. 1a and b) with a uniform size (Fig. 1d and e). The ZTC@M nanoparticle was covered with cytomembrane as seen through the TEM (Fig. 1c). Additionally, the surface was observed to be slightly smoother than ZTC (Fig. 1b and d). The size was also bigger than ZTC (Fig. 1d and f), with an increase in size reported from $175 \mathrm{~nm}$ to $253 \mathrm{~nm}$. Besides, the comparison with the surface potential before modification of AGS cytomembrane showed a reversal from positive $13.8 \mathrm{mV}$ to negative $-16.4 \mathrm{mV}$, indicating successful coating of AGS cytomembrane (Fig.1h). This result was consistent with the gel electrophoresis result (Fig. 1g). Also, the stability of ZTC@M was evaluated, where the results showed that the size did not change in ultrapure water within a week (Fig. 1i), indicating that the nanoparticle was stabilized.

The absorption peaks of TPZ (470 nm) and Ce6 (400 and $660 \mathrm{~nm})$ were presented in the absorption spectrum of ZTC@M (Fig. 1j), indicating successful loading of the nanoparticle with TPZ and Ce6. Simultaneously, the loading capacities of $\mathrm{Ce} 6$ and TPZ were calculated, which were found to be $77.57 \pm 0.48 \%$ and $53.86 \pm 3.48 \%$, respectively. The results illustrated that the frame ZIF-8 could co-load both TPZ and Ce6 for the cooperative therapy.

DPBF is a typical analytical reagent of ROS and is used to analyze the production of ROS. Compared to the control groups, the remaining DPBF in the ZTC@M group irradiated with the US was found to be significantly lower (Fig. 1k), indicating that ZTC@M upon US irradiation could produce much more ROS from Ce6.

The CCK-8 assay was conducted to explore the best working concentration of this nanoparticle. When the loading content of TPZ reached $8 \mathrm{mg}$, the viability of the cells was inhibited down to 40\% (Fig. 2a), determining the quantity of TPZ to be loaded as $8 \mathrm{mg}$. Moreover, ZIF-8 and Ce6 showed no obvious cytotoxicity to AGS cells (Fig. 2b). The cytotoxicity of nanoparticles was found to be weaker than that of a single TPZ drug (Fig. 2b), suggesting a weakening of the toxicity of the single drug by the nanoparticle. Also, no cytotoxicity was observed with the concentration of nanoparticles being up to $30 \mu \mathrm{g} / \mathrm{mL}$ (Fig. $2 \mathrm{~b})$. Thus, the working concentration of the nanoparticle was chosen as $30 \mu \mathrm{g} / \mathrm{mL}$.

The hemolysis assay was conducted to analyzed the biocompatibility of ZTC@M. The hemolysis rate below $10 \%$ was considered safe for intravenous injection[31].The hemolysis rate was observed to be less than $1 \%$ when the concentration of ZTC@M used was up to $300 \mu \mathrm{g} / \mathrm{mL}$, which was an acceptable range for intravenous administration (Fig. $2 \mathrm{c}$ and $2 \mathrm{~d}$ ), indicating good biocompatibility.

The drug-releasing ability of TPZ and Ce6 from ZTC@M was evaluated in PBS at two different pH. Comparing with $\mathrm{pH}$ 7.4, the release rate was increased in the solution having pH 5.5 (Fig. 2e and f) due to 
its $\mathrm{pH}$-responsive decomposition ability. This illustrated an increased amount of release of Ce6 and TPZ in the PBS solution with $\mathrm{pH} 5.5$. Incubation for $6 \mathrm{~h}$ at $\mathrm{pH} 5.5$ released approximately $81.49 \pm 1.58 \%$ of loaded TPZ and $62.20 \pm 0.55 \%$ of loaded Ce6 from the ZTC@M, and these values were much higher than the values obtained at $\mathrm{pH}$ 7.4. Furthermore, the releasing ability of Ce6 from ZTC@M was evaluated with/without the US. It was found that the release rates were higher when irradiated with the US than the ones without the US irradiation (Fig. 2e). After the treatment with the US at pH 5.5, 78.18 $\pm 0.86 \%$ of loaded Ce6 was released from ZTC@M. Thus, in cooperation with the US, the pH responsiveness of ZTC@M was applied to release drugs in an acidic solution to attain a combined target treatment.

\section{Cellular Uptake}

The results of the cell fluorescence showed that compared to the incubation at pH 7.4, AGS cells showed higher fluorescence intensities at pH 5.5 (Fig. 3a and c). After the addition of cytomembrane of AGS, stronger fluorescence intensities were presented in the ZTC@M group, comparing wirh the ZTC group. Further, the signal intensities from AGS cells wrapped with cytomembrane of other species cells (4T1, mouse breast cancer cell) showed weaker signals than the cells wrapped with a homologous tumor cytomembrane (Fig. 3a and C). The results of the flow cytometer were consistent with the results of the cell fluorescence (Fig. 3b and d). Nanoparticles can be better degraded and released in the site of the microenvironment, which is similar to the tumor acidic microenvironment, Also, free Ce6 can easily enter the cells in such case. Additionally, because of the homologous cytomembrane, more nanoparticles can be taken up by the cells. All of these indicate that ZTC@M especially recognizes homologous tumor cytomembrane and makes use of the tumor acidic microenvironment to target the release of the drug and achieve anti-tumor function.

\section{In vitro ROS/Hypoxia assay}

Intracellular ROS levels were evaluated by DCFH-DA, whose green fluorescence was enhanced with the increase in ROS level inside the cells. As presented in Fig. 4a and e, the intensity of ROS green fluorescence in the group irradiated by the US (ZTC@M+US) was found to be much higher than that found in ZC, ZTC, and ZTC@M groups without the US ( $<<0.0001)$, which indicated that Ce6 irradiated by the US released ROS. The reason behind this could be attributed to Ce6 being a sonosensitizer, which may produce ROS after the treatment with the US.

Next, the ability to induce hypoxia by ROS was evaluated by the hypoxia reagent. The Image $-\mathrm{iT}^{\mathrm{TM}}$ Green Hypoxia Reagent is an end-point assay reagent whose signal increases with the reduced oxygen levels. The green fluorescence is enhanced with the increase in hypoxia level. Our results showed that the green fluorescence in the CON group was weaker than that of the ZTC $(p=0.0068), \mathrm{ZTC} @ M(p=0.0034)$, and ZTC@M+US $(P<0.0001)$ groups (Fig. 4b and f). Meanwhile, the strongest green fluorescence was seen in the ZTC@M+US group, which was even stronger than that of the ZTC@M group ( $P<0.0001)$ (Fig. 4b and 
f). This can be explained by the increased hypoxia in cells released by Ce6 due to the accumulation of ROS. Also, the release of ROS and increased hypoxia in cells is enhanced by the US.

HIF-1a is considered as a key mediator of signal in poorly oxygenated areas and can stay steady under hypoxia environment [32,33]. In normoxia conditions, HIF-1a is always inactivated and degraded hence, it is hardly detected [34]. HIF-1a can stay stabilized in poor oxygen conditions and can be translocated to the nucleus responding rapidly to oxygen deprivation [35]. Further, IF and WB of HIF-1a were performed to demonstrate the reduction of intracellular hypoxia by ROS consumption. With an increase in hypoxia level, the green fluorescence of HIF-1 a could be strengthened. It was found that the green fluorescence observed in the ZTC@M+US group was stronger than any other group, i.e., CON, ZC, ZT ZTC, and ZTC@M groups ( $P<0.0001$ ) (Fig. 4c and g), showing that the hypoxia level of the ZTC@M+US group was the strongest. In Fig. 4d and h, the levels of HIF-1a protein in the ZTC@M+US group were found to be much higher than that of the CON group $(P<0.0001)$ and ZTC@M+US group $(P<0.05)$, which was in accord with the results of IF. These results proved that the ROS released from Ce6 could induce intracellular hypoxia, which provided a hypoxic environment to TPZ.

\section{In vitro therapeutic effect}

To evaluate the killing effect of nanoparticles, Calcein/PI staining and apoptosis assays were conducted. Fig. 5a showed that the red fluorescent intensities from dead cells in the ZTC group were stronger than that found in the ZT and ZC groups $(P<0.05)$, indicating that the efficiency of nanoparticles was better in combination with $\mathrm{Ce} 6$ and TPZ than that of a single drug. Meanwhile, the red fluorescence intensities of the cytomembrane target group (ZTC@M) were higher than those groups without cytomembrane (ZTC) $(P<0.05)$. This was because the homologous AGS cytomembrane specifically increased the uptake of nanoparticles by AGS cells and enhanced the killing effect on tumor cells. Also, after the US treatment, a much stronger red fluorescence was seen in most of the cells. The quantitative analysis of fluorescence presented that the therapeutic effect of the SDT treatment group (ZTC@M+US) was found to be higher than that of the group without the SDT (ZTC@M) (Fig. 5a and c) $(P<0.05)$.

Similar results were shown by the apoptosis assay (Fig. $5 b$ and d). The percentages of apoptotic cells in CON, ZC, ZT, ZTC, ZTC@M, and ZTC@M+US groups were approximately 8.13\%, 17.40\%, 15.49\%, 24.10\%, $49.87 \%$, and 72.77 , respectively. There was a significant difference observed between ZTC and ZT/ZC. A higher rate of apoptosis was observed in ZTC@M compared to that of the ZTC. The highest apoptosis rate was demonstrated in the group treated with US (ZTC@M+US). All these results illustrated that targeting the drug along with SDT treatment could enhance the killing effect on tumor cells.

\section{In vivo biocompatibility research}

To verify the safety in vivo, we evaluated the biocompatibility of nanoparticles. Fig. $6 a$ shows that there were no significant differences in the counts of white blood cell (WBC), red blood cell (RBC), hemoglobin 
$(\mathrm{Hb})$, and platelet (PLT) between the groups observed on 7/30 $\mathrm{d}$ and in the control group, illustrating that there was no significant blood toxicity found in ZTC@M in vivo. Meanwhile, aspartate aminotransferase (AST), alanine aminotransferase (ALT), alkaline phosphatase (ALP), and blood urea nitrogen (BUN) were detected to study the presence of any liver and kidney damage. As shown in Fig. 6a, no significant differences were observed in these indexes in the three groups. Also, to confirm whether nanoparticles can induce organ damage, the H\&E staining of organs was compared among the three groups. The results demonstrated that no apparent damage or pathological changes were observed in the experimental groups, which showed consistency with the control group (Fig. 6b). Hence, these results presented that ZTC@M had good biocompatibility with no apparent toxicity in vivo, indicating it to be a potential nanoparticle for in vivo anti-tumor experiments.

\section{In vivo SDT therapy}

Nude mice bearing AGS tumors were used as the model to investigate the anti-tumor effect of SDT therapy of ZTC@M. As shown in Fig. 7b, there was no significant loss of body weight in mice among all six groups during the treatment, which demonstrated that the nanoparticles had good biocompatibility with no evident toxicity to mice. It was observed that the growth of tumors treated with PBS was a little more rapid than those treated with ZC and ZT (Fig. 7c), indicating that single-drug therapy had limitations. Simultaneously, Fig. 7c shows that the growth of tumors was slightly slower in the group treated with nanoparticles combined with Ce6 and TPZ than that of a single drug, which showed that combined therapy worked relatively better than a single drug that had limited efficiency. However, the growth of the tumor in the targeting group (ZTC@M) was further reduced, and the inhibition of tumor growth was found to be stronger than the previous three groups, but it still could not reduce the growth of the tumor completely. The tumor growth was inhibited in the ZTC@M mice treated with the US irradiation compared to those without the US, indicating a significant anti-tumor efficiency by the US. Regarding the tumor inhibition ratio (Fig. 7d), the inhibition rate reached up to approximately 87\% in the ZTC@M+US group, which was higher than any other group, indicating the same results as the tumor volume. The reasons behind these results might be explained as follows: firstly, the nanoparticles encapsulated with homologous tumor cytomembrane enhanced its targeting ability and were recognized easily by tumor cells; hence, more nanoparticles could enter tumor cells and perform the anti-tumor function. However, without US irradiation, Ce6 could not be activated completely, and the function was limited. Secondly, after irradiation with the US, the accumulation of ROS released by Ce6 could aggravate hypoxia in the tumor microenvironment and kill tumor cells. Finally, hypoxia further activated the anti-tumor effect of TPZ and induced the death of tumor cells. These results demonstrated that ZTC@M+US could efficiently inhibit tumors and exert anti-tumor function.

Further, H\&E staining of the tumor was performed to evaluate the therapeutic efficacy. An obvious necrotic tissue was observed in the tumors of mice treated with the ZTC@M+US group compared to the other groups (Fig. 7e). Further, the TUNEL staining was performed to observe the tumor injure in mice among the different groups (Fig. 7e). The results indicated that there was a small number of apoptotic 
cells observed in the tumors of PBS, ZC, ZT, and ZTC groups. In the ZTC@M group, many apoptotic cells were seen, but the number was less than that of the ZTC@M+US group. These results were in accord with the results of H\&E staining.

\section{ZTC@M induced ROS contributed to AGS pyroptosis}

Some studies reported that the ROS contributed to apoptosis and pyroptosis[36, 37].Pyroptosis is different from cell apoptosis and is an inflammatory form that mediates the programmed death of cells[38].It is activated by caspase-1/4/5/11 and can lead to cell damage, including fragmentation of chromatin, cell swelling, lysis of plasma membranes, and release of intracellular proinflammatory contents[39, 40].It is well known that caspase-1, NLRP3 (NACHT, LRR, and PYD domain-containing protein $3)$, and ACS molecules can induce pyroptosis in tumor cells. Caspase-1 is the activator of pyroptosis, which can induce the release of inflammatory factors, i.e., interleukin-1 beta (IL-1 3 ) and interleukin-18 (IL18)[41].ACS comprises a caspase recruitment domain and a pyrin domain and is the adaption protein of NLRP3[42].The NLRP3 inflammasome, an inflammatory protein complex, consists of the sensory molecule NLRP3, ACS, and caspase-1, which is the most characterized inflammasome[43, 44].It is also well known that ROS is an activator of NLRP3 inflammasome[45, 46].

To determine if the nanoparticles could induce pyroptosis, the levels of these proteins were detected by western blotting. The ZTC@M groups exhibited higher levels of caspase-1, NLRP3, and ACS compared to that of the control groups (Fig. 8a and b, all P-value<0.05). Comparing with the control and ZTC@M groups, the levels of caspase-1, NLRP3, and ACS in the ZTC@M+US group were found to be significantly higher (Fig. 8a and b, all p-value<0.05). It demonstrated that caspase 1, caspase 3, GSDMD, NLRP3, and ACS were all upregulated after being irradiated by the US. These results showed that the ROS produced by the ZTC@M group treated with the US could induce pyroptosis in AGS cells, which enabled it to play the anti-tumor function.

Cells undergoing pyroptosis show distinct morphological features. Deron R. Herr et al. proved that the most prominent pyroptotic cells represented cell swelling, the retraction of cellular processes, and the emerging of pores in the cell surface [47]. AGS cells were also evaluated for detailed surface morphology by phase-contrast microscopy (Fig. 8c and d) after the ZTC@M+US treatment. Untreated AGS cells appeared normal under microscopy showing characteristics such as extended processes among the cells (Fig. 8c). After the treatment with ZTC@M+US, it was found that the loss of processes and cell swelling manifested as a significant increase in the cell size (Fig. 8d). Further, the surface morphology was observed by the SEM, and the most prominent treated cells were found in the numerous pits or pores of different sizes across the cell surface (Fig. 8f), which was correlated with collapsing of the structure and flattening of the cell shape (Fig. 8e). Simultaneously, it also presented a rounded morphology due to the complete retraction of cellular processes (Fig. 8e). Hence, proving that our nanoparticles could induce cell pyroptosis. 


\section{Conclusion}

ZIF-8 showed a good Ce6 and TPZ loading capacity because of its high surface area structure, good cellular release efficiency, and responsiveness to an acidic environment. The US irradiation allowed ZTC@M to consume the intracellular oxygen and generate ROS, which enhanced intracellular hypoxia to activate TPZ, ultimately enabling it to kill the cancer cells. Our results demonstrated that ZTC@M activated NLRP3, caspase-1, and ACS by generating ROS through SDT, leading to pyroptosis of gastric cancer cells. In summary, we successfully synthesized a US-activated, $\mathrm{pH}$-sensitive, hypoxia-induced ZIF8 organic-metal framework that targeted the delivery of TPZ and Ce6 to be used as a combination of SDT and chemotherapy, thus, enhancing the anti-cancerous effect of the drug, which may provide a potential method for gastric cancer therapy.

\section{Materials And Methods}

\section{Drugs}

Zinc nitrate hexahydrate $\left(\mathrm{Zn}\left(\mathrm{NO}_{3}\right)_{2} \cdot 6 \mathrm{H}_{2} \mathrm{O}\right)$, 1,2-dimethylimidazole (1,2-MIL), and 1,3-diphenyl isobenzofuran (DPBF) were purchased from Aladdin (China). TPZ was purchased from MCE (US), while Ce6 was purchased from Frontier Scientific (US). The primary antibodies Caspase-1, ACS, NLRP3 were purchased from Proteintech (China). Hypoxia-inducible factor 1-alpha (HIF-1a) and GAPDH were purchased from CST (US).

\section{Synthesis of ZIF-8@TPZ/Ce6 (ZTC)}

Solution $A$ and $B$ were prepared by dissolving $300 \mathrm{mg}$ of $\mathrm{Zn}\left(\mathrm{NO}_{3}\right)_{2} \cdot 6 \mathrm{H}_{2} \mathrm{O}(\mathrm{A})$ and $170 \mathrm{mg}$ of $1,2-\mathrm{MIL}(\mathrm{B})$ in methanol $(40 \mathrm{ml})$ separately. Then, solution $B$ was added to $A$ and stirred thoroughly in a flask for 2 min. Next, TPZ and Ce6 $(1 \mathrm{mg} / 200 \mathrm{u})$ methanol solutions were added to the solution and stirred continuously for $5 \mathrm{~min}$. After allowing it to stand for $4 \mathrm{~h}$, the solution was centrifuged at $12000 \mathrm{rpm}$ for $10 \mathrm{~min}$, followed by two steps of washing with methanol and DMSO. This solution was then freeze-dried. Similarly, ZIF-8@Ce6 (ZC) and ZIF-8@TPZ (ZT) were synthesized following the same procedure. The loading content of Ce6/TPZ was calculated as follows: loading content of TPZ/ Ce6 = (Quality of input Ce6/TPZ-Quality of TPZ/ Ce6 in the supernatant)/Quality of input TPZ/ Ce6 $\times 100 \%$.

\section{Synthesis of ZIF-8@Ce6/TPZ@ cytomembrane (ZTC@M)}

Membrane Protein Extraction Kit was used to extract the cytomembrane of the AGS cell. The collected cytomembrane was added to cytosol extraction buffer (with phenylmethanesulfonyl fluoride, PMSF) and cooled in an ice bath for $10 \mathrm{~min}$. Next, the solution was freeze-thawed repeatedly in liquid nitrogen and room temperature. The solution was then centrifuged at $700 \mathrm{~g}\left(10 \mathrm{~min}, 4^{\circ} \mathrm{C}\right)$. The collected supernatant was centrifuged $\left(4^{\circ} \mathrm{C}, 14000 \mathrm{~g}, 30 \mathrm{~min}\right)$, and the cytomembrane fragment was collected from the 
precipitate. ZTC ( $2 \mathrm{mg} / \mathrm{mL}, 1 \mathrm{ml})$ was added to the cytomembrane fragment of AGS $(4 \mathrm{mg} / \mathrm{mL}, 1 \mathrm{ml})$ and stirred for $2 \mathrm{~h}$ at $4{ }^{\circ} \mathrm{C}$. After repeatedly using a liposome extruder (membrane of polycarbonate porous, $400 \mathrm{~nm})$, the spare cytomembrane fragments were discarded via centrifugation $\left(4^{\circ} \mathrm{C}, 12000 \mathrm{~g}, 10 \mathrm{~min}\right)$. Finally, the ZIF-8@Ce6/TPZ@ cytomembrane (ZTC@M) was acquired. Following the same procedure, we obtained the cytomembrane of breast cancer 4T1 cell and synthesized ZTC@ cytomembrane 4T1 (ZTC@m).

\section{Gel Electrophoresis}

The concentration of AGS cytomembrane and ZTC@M were detected using the BCA Protein Assay Kit (Beyotime, China). All prepared protein samples were loaded onto the $10 \%$ sodium dodecyl sulfatepolyacrylamide gels (SDS-PAGE) for separation. Coomassie Blue (Beyotime, China) was used to stain the protein, and the picture was taken after destaining the gel with water for $6 \mathrm{~h}$.

\section{In vitro drug release at different $\mathrm{pH}$}

ZTC@M $(2 \mathrm{mg} / \mathrm{mL})$ was dissolved in PBS at pH 5.5 and 7.4, respectively, and put in a constant temperature shaker (Thermo Fisher Scientific, USA) at $37^{\circ} \mathrm{C}$ for $6 \mathrm{~h}$. The solution $(500 \mu \mathrm{L})$ was collected and centrifuged (12000 rpm, $10 \mathrm{~min}$ ) at seven time points $(0,1,2,3,4,5$ and $6 \mathrm{~h})$, and equal dosages of PBS were replenished. The absorbance of TPZ/Ce6 in the supernatant was determined at $470 \mathrm{~nm}$ (TPZ) and $660 \mathrm{~nm}$ (Ce6), respectively. Each group was designed to have three parallel samples. The release rate of TPZ/Ce6 was calculated as follows: release rate of TPZ/Ce6 = Total quantity of released drug/ Total quantity of TPZ/Ce6 $\times 100 \%$.

\section{Production of Reactive Oxygen Species (ROS)}

The production of ROS by ZTC@M was determined by 1,3-Diphenyl isobenzofuran (DPBF). The absorbance values of mixture DPBF and ZTC@M were measured using the US at $410 \mathrm{~nm}$ per minute. The other two groups, i.e., the DPBF and DPBF + ZTC@M groups were set as the control groups. The remaining quantity of DPBF was calculated as follows: 100 - (Initial absorbance - Final absorbance)/Initial absorbance $\times 100 \%$.

\section{In vitro Cytotoxicity Assay}

CCK-8 assay was performed to determine the cytotoxicity against the AGS cells. AGS cells were incubated in a 96-well plate and cultured overnight. Then, different concentrations of ZTC@M (10, 20, 30, 40, 50 $\mu \mathrm{g} / \mathrm{mL}$ ) were added to each well and cultured for $24 \mathrm{~h}$. Each group had five parallel samples. After washing with PBS, $100 \mu \mathrm{L}$ of the medium was added to each well along with $10 \mu \mathrm{L}$ of CCK-8 solution. 
After incubated for $1 \mathrm{~h}$, the absorbance of plate was detected at $450 \mathrm{~nm}$ by a microplate reader, and the cell survival rate was calculated.

\section{Hemolysis Assay}

The hemolysis reaction was used to analyze the blood compatibility of ZTC@M nanoparticles. The fresh blood was collected from mice and washed repeatedly with PBS to obtain red blood cells (RBC). Different concentrations of nanoparticles with a constant volume of 500uL PBS were added to 500uL of RBC solution. Each group was designed with three parallel samples, where deionized water and PBS were positive control (PC) and negative (NC) control, respectively. After further incubation at $37^{\circ} \mathrm{C}$ for $4 \mathrm{~h}$, all samples were centrifuged. The absorption of the supernatant was determined at $540 \mathrm{~nm}$ by the VARIOSKAN LUX microplate reader. The Hemolysis rate was calculated: Hemolysis rate $(\%)=\left(O D_{\text {test }}{ }^{-}\right.$ $\left.\mathrm{OD}_{\mathrm{NC}}\right) /\left(\mathrm{OD}_{\mathrm{PC}}-\mathrm{OD}_{\mathrm{NC}}\right) \times 100 \%$.

\section{Cellular Uptake}

AGS cells $\left(1 \times 10^{5} /\right.$ well) were seeded in a confocal dish and incubated overnight. The confocal dishes were seeded with Opti-MEM medium containing ZTC@M (TPZ $12 \mu \mathrm{g} / \mathrm{mL}$ and Ce6 $2 \mu \mathrm{g} / \mathrm{mL}$ ), which was cultured for $4 \mathrm{~h}$ at $\mathrm{pH}$ 7.4/5.5. The cells were then washed with PBS thrice and stained with DAPI and imaged using laser confocal scanning microscopy (LCSM).

Additionally, to analyze the uptake of ZTC@M in different groups, the FACSVerse flow cytometry (BD, USA) was used to analyze the Ce6 fluorescence intensity (FL) of AGS cells.

\section{In vitro detection of ROS}

In vitro, 2,7-dichlorodihydrofluorescein diacetate (DCFH-DA, Beyotime, China) was used to measure the ROS level. AGS cells were seeded in a 6-well plate and incubated for $24 \mathrm{~h}$. Opti-MEM medium containing ZTC@M was added to cultured cells and incubated for $4 \mathrm{~h}$ in the dark. The medium was then removed and washed thrice using PBS. The cells were then incubated with DCFH-DA for $30 \mathrm{~min}$, and the fluorescence imaging of AGS cells was conducted by using fluorescence microscopy while one group was irradiated by the US (1.0 MHz, $\left.1.5 \mathrm{~W} / \mathrm{cm}^{2}, 3 \mathrm{~min}\right)$.

\section{In vitro detection of hypoxia and immunofluorescence (IF)}

Image-iT $\mathrm{T}^{\mathrm{TM}}$ Green Hypoxia Reagent was used to detect the oxygen concentration in vitro. AGS cells were seeded in the confocal dish and incubated for $24 \mathrm{~h}$. After incubating the cells with different nanoparticles for $4 \mathrm{~h}$ and irradiating them with the US $\left(1.0 \mathrm{MHz}, 1.5 \mathrm{~W} / \mathrm{cm}^{2}, 3 \mathrm{~min}\right)$, cells were washed thrice with PBS. 
Then, Image $-\mathrm{iT}^{\mathrm{TM}}$ Green Hypoxia Reagent stock solution $(5 \mu \mathrm{M})$ was added to the cells, and then incubated for $30 \mathrm{~min}$. The cells were then stained with DAPI for $10 \mathrm{~min}$ and imaged using LCSM.

Cells were inoculated on the coverslip. After treatment with nanoparticles, cells were fixed with $4 \%$ paraformaldehyde for $15 \mathrm{~min}$. Successively, 0.1\% Triton X-100 and 10\% BSA were used to permeabilize and block cells. The primary antibody HIF-1a (1:500, CST, US) was added to cells and incubated overnight at $4{ }^{\circ} \mathrm{C}$. Subsequently, cells were incubated with Alexa Flour 488 goat anti-rabbit secondary antibody (1:500, Beyotime, China) for 30 min. Finally, DAPI was used to label the nuclei, and the images were captured by an inverted microscope.

\section{Cell apoptosis}

The flow cytometry assay was used to evaluate the apoptosis of AGS cells. AGS cells $\left(3 \times 10^{5}\right.$ cells/well) were seeded into a 6-well plate and incubated for $24 \mathrm{~h}$. After washing the cells thrice with PBS, Opti-MEM medium containing ZC, ZT, ZTC, and ZTC@M, were added to the plates, respectively, and cultured for $4 \mathrm{~h}$. An equal concentration of Ce6 and TPZ was used in each group (TPZ $12 \mu \mathrm{g} / \mathrm{mL}$ and Ce6 $2 \mu \mathrm{g} / \mathrm{mL}$ ). Especially, after initially incubating the plates for $2 \mathrm{~h}$, one plate with ZTC@M was irradiated by the US (1.0 $\mathrm{MHz}, 1.5 \mathrm{~W} / \mathrm{cm}^{2}, 3 \mathrm{~min}$ ) and continued for incubation for another $2 \mathrm{~h}$. Next, cells were digested with EDTA-free trypsin and centrifuged (5 min, 1000rpm). The cells were mixed with $500 \mu \mathrm{L}$ of binding Buffer, to which $5 \mu \mathrm{L}$ of Annexin V-FITC and $10 \mu \mathrm{L}$ of PI were added and incubated for $5 \mathrm{~min}$ in the dark. Finally, the cell apoptosis was detected using FACSVerse flow cytometry (BD, USA).

\section{Live/Dead cell staining}

Calcein/PI Live/Dead Viability/Cytotoxicity Assay Kit (Beyotime, China) was applied to analyze the live/dead cell staining of AGS. The AGS cells $\left(1 \times 10^{5}\right.$ cells/well) were seeded into a 12 -well plate and incubated for $24 \mathrm{~h}$. After washing the cells thrice with PBS, the Opti-MEM medium containing ZC, ZT, ZTC, and ZTC@M was added to the cells, respectively. One plate with ZTC@M was irradiated using US (1.0 $\mathrm{MHz}, 1.5 \mathrm{~W} / \mathrm{cm}^{2}, 3 \mathrm{~min}$ ) and incubated initially for $2 \mathrm{~h}$ with further incubation of $2 \mathrm{~h}$. After culturing the cells for $4 \mathrm{~h}, 1 \mathrm{~mL}$ of detecting buffer was added to the cells along with $1 \mu \mathrm{L}$ of Calcein and $1 \mu \mathrm{L}$ of PI and incubated for $30 \mathrm{~min}$ to stain the live and dead cells. The fluorescence of AGS cells was observed under a fluorescence microscope.

\section{Western blot}

AGS cells $\left(3 \times 10^{5}\right.$ cells/well) were seeded into a 6 -well plate. Once the cells reached approximately $80 \%$ confluence, TPZ $(12 \mu \mathrm{g} / \mathrm{mL})$ and ZTC@M $(20 \mu \mathrm{g} / \mathrm{mL})$ were added to each well and incubated for $24 \mathrm{~h}$. After incubating the plates for $4 \mathrm{~h}$, one plate with ZTC@M was irradiated using US $\left(1.0 \mathrm{MHz}, 1.5 \mathrm{~W} / \mathrm{cm}^{2}, 3\right.$ min), and then was further incubated. Next, the cells were lysed with RIPA buffer with PMSF. All protein 
samples were loaded onto the $10 \%$ SDS-PAGE and then transferred onto a polyvinylidene fluoride (PVDF) membrane. After incubating the membranes with blocking solution for $30 \mathrm{~min}$, the PVDF membranes were incubated with primary antibodies against caspase-1 (1:500), ACS (1:500), NLRP3 (1:500), hypoxiainducible factor 1-alpha (1:1000), and GAPDH (1:10000) overnight, respectively. The membranes were then washed thrice with TBST, and incubated with secondary antibodies. Finally, the results were visualized using the Pierce ECL Western Blotting Substrate.

\section{In vivo safety evaluation}

The female nude mice (5 weeks) were randomly divided into three groups. Two groups of mice were injected with $200 \mu \mathrm{L}$ of ZTC@M (10 mg/kg Ce6 and $40 \mathrm{mg} / \mathrm{kg}$ TPZ) every $3 \mathrm{~d}$ for 3 times through the tail vein. The other group was used as the control, which was injected with an equal amount of PBS. The mice were sacrificed after $7 \mathrm{~d}$ and $30 \mathrm{~d}$, respectively. Later, mice blood was collected and analyzed. Also, organs (heart, liver, spleen, lung, and kidney) were collected and stained for H\&E.

\section{In vivo therapy}

All the animal experiments were conducted strictly under the guidelines of the National animal management regulations of China and were approved by the Animal Experimental Ethics Committee of Guangxi Medical University.

The nude mice bearing AGS tumors were used as the model, and the anticancer effect of ultrasound therapy of nanoparticles was analyzed. When the tumors grew up to $80-100 \mathrm{~mm}^{3}$, the AGS tumorbearing nude mice were divided randomly into six groups, with each group having five parallel samples: (1) PBS, (2) PBS + ZC, (3) PBS + ZT, (4) PBS + ZTC, (5) PBS + ZTC@M, and (6) PBS + ZTC@M + the US. PBS containing different nanoparticles $(200 \mu \mathrm{L}, 10 \mathrm{mg} / \mathrm{kg}$ Ce6, and $40 \mathrm{mg} / \mathrm{kg}$ TPZ) were injected into the mice via tail vein every $3 \mathrm{~d}$ for 3 times, and US $\left(1.5 \mathrm{~W} / \mathrm{cm}^{2}, 3 \mathrm{~min}\right)$ was used to irradiate the group (6) after $6 \mathrm{~h}$. The mice were observed for $20 \mathrm{~d}$. The body weight and tumor volume were recorded every $2 \mathrm{~d}$, and tumor volume was calculated as follows: Tumor volume $=\left(\right.$ tumor length $\times$ tumor width $\left.{ }^{2}\right) / 2$. All mice were sacrificed at the end of observation, and the tumors were collected followed by HE staining and TUNEL staining.

\section{Statistical analysis}

All data were presented as the mean \pm standard deviation (SD). The student's t-test was used to analyze the difference between the two groups. Prism 6 software (GraphPad 6) was used for all the statistical analyses. $P<0.05$ was considered statistically significant.

\section{Declarations}




\section{Acknowledgements}

We acknowledge the support from the National Natural Science Foundation of China (No. 8206043), the Natural Science Foundation of Guangxi (No.2017AB45153, No.2018GXNSFAA294111 and No.2020GXNSFBA159068); the Guangxi Key Research and Development Project (No. AB18126058); the Guangxi Key Scientific Research and Technology Development Project (No.1598011-4); the Science and Technology Plan Project of Qingxiu District of Nanning, Guangxi (No.2019023); the Innovation Project of Guangxi Graduate Education (No. YCBZ2021054); and “Medical Excellence Award” Founded by the Creative Research Development Grant from the First Affiliated Hospital of Guangxi Medical University.

\section{Authors' contributions}

Junqiang Chen developed the concept and supervised the project. Zhu Yu and Wenlong Cao synthesize the nanoparticle and conducted the in vitro experiments. Zhu Yu, Wenlong Cao and Chuangye Han wrote the paper. Yue Qiu, Jiancheng Wang, Siwen Zhang and Senfeng Liu were responsible for the animal experiments. Junfu Wang, Zhen Wang, and Shutian Mo analyzed the data and prepared the figures. All authors have given approval to the final version of the manuscript.

\section{Funding}

The study was supported by the National Natural Science Foundation of China (No. 8206043), the Natural Science Foundation of Guangxi (No.2017AB45153, No.2018GXNSFAA294111 and No.2020GXNSFBA159068); the Guangxi Key Research and Development Project (No. AB18126058); the Guangxi Key Scientific Research and Technology Development Project (No.1598011-4); the Science and Technology Plan Project of Qingxiu District of Nanning, Guangxi (No.2019023); the Innovation Project of Guangxi Graduate Education (No. YCBZ2021054); and "Medical Excellence Award" Founded by the Creative Research Development Grant from the First Affiliated Hospital of Guangxi Medical University.

\section{Availability of data and materials}

The authors declare that the data related to this study are provided upon request.

\section{Ethical approval and consent to participate}

All the animal experiments were conducted strictly under the guidelines of the National animal management regulations of China and were approved by the Animal Experimental Ethics Committee of Guangxi Medical University. 


\section{Consent for publication}

Not Applicable

\section{Competing interests}

There are no conflicts to declare.

\section{References}

1. Sung H, Ferlay J, Siegel RL, Laversanne M, Soerjomataram I, Jemal A, Bray F. Global cancer statistics 2020: GLOBOCAN estimates of incidence and mortality worldwide for 36 cancers in 185 countries. CA Cancer J Clin 2021.

2. Smyth EC, Nilsson M, Grabsch HI, Grieken NCv, Lordick F. Gastric cancer. Lancet. 2020;396:14.

3. Song Z, Wu Y, Yang J, Yang D, Fang X. Progress in the treatment of advanced gastric cancer. Tumor Biology. 2017;39(7):7.

4. Johnston FM, Beckman M. Updates on Management of Gastric Cancer. Curr Oncol Rep. 2019;21(8):67.

5. Correa P. Gastric cancer: overview. Gastroenterol Clin North Am. 2013;42(2):211-7.

6. Rengeng L, Qianyu Z, Yuehong L, Zhongzhong P, Libo L. Sonodynamic therapy, a treatment developing from photodynamic therapy. Photodiagnosis Photodyn Ther. 2017;19:159-66.

7. Bhatta A, Krishnamoorthy G, Marimuthu N, Dihingia A, Manna P, Biswal HT, Das M, Krishnamoorthy G. Chlorin e6 decorated doxorubicin encapsulated chitosan nanoparticles for photo-controlled cancer drug delivery. Int J Biol Macromol. 2019;136:951-61.

8. Li Q, Liu Q, Wang P, Feng X, Wang H, Wang X. The effects of Ce6-mediated sono-photodynamic therapy on cell migration, apoptosis and autophagy in mouse mammary 4T1 cell line. Ultrasonics. 2014;54(4):981-9.

\section{Light-triggered theranostic liposomes for tumor diagnosis and combined.}

10. Liu Y, Ma K, Jiao T, Xing R, Shen G, Yan X. Water-Insoluble Photosensitizer Nanocolloids Stabilized by Supramolecular Interfacial Assembly towards Photodynamic Therapy. Sci Rep. 2017;7:42978.

11. Guo D, Xu S, Yasen W, Zhang C, Shen J, Huang Y, Chen D, Zhu X. Tirapazamine-embedded polyplatinum(iv) complex: a prodrug combo for hypoxia-activated synergistic chemotherapy. Biomater Sci. 2020;8(2):694-701.

12. Marcu L, Olver I. Tirapazamine From Bench to Clinical Trials. Current Clinical Pharmacology. 2006;1:9.

13. Reddy SB, Williamson SK. Tirapazamine a novel agent targeting hypoxic tumor cells. Expert Opin Investig Drugs. 2009;18(1):11. 
14. Gandara DR, Lara PN Jr, Goldberg Z, Le QT, Mack PC, Lau DH, Gumerlock PH. Tirapazamine: prototype for a novel class of therapeutic agents targeting tumor hypoxia. Semin Oncol. 2002;29(1 Suppl 4):102-9.

15. Gatzemeier U, Rodriguez G, Treat2 J, Roemeling Rv VM, Viallet J, Rey A. Tirapazamine cisplatin: the synergy. Br J Cancer. 1998;77:3.

16. Denny WA, Wilson WR. Tirapazamine: a bioreductive anticancer drug that exploits tumour hypoxia. Exp Opin Invest Drugs. 2000;9(12):3.

17. Lu G, Li S, Guo Z, Farha OK, Hauser BG, Qi X, Wang Y, Wang X, Han S, Liu X, et al. Imparting functionality to a metal-organic framework material by controlled nanoparticle encapsulation. Nat Chem. 2012;4(4):310-6.

18. Wu X, Yang C, Ge J, Liu Z. Polydopamine tethered enzyme/metal-organic framework composites with high stability and reusability. Nanoscale. 2015;7(45):18883-6.

19. Cai W, Wang J, Chu C, Chen W, Wu C, Liu G. Metal-Organic Framework-Based Stimuli-Responsive Systems for Drug Delivery. Adv Sci (Weinh). 2019;6(1):1801526.

20. Cheng C, Li C, Zhu X, Han W, Li J, Lv Y. Doxorubicin-loaded Fe304-ZIF-8 nano-composites for hepatocellular carcinoma therapy. J Biomater Appl. 2019;33(10):1373-81.

21. An J, Hu YG, Li C, Hou XL, Cheng K, Zhang B, Zhang RY, Li DY, Liu SJ, Liu B, et al. A pH/Ultrasound dual-response biomimetic nanoplatform for nitric oxide gas-sonodynamic combined therapy and repeated ultrasound for relieving hypoxia. Biomaterials. 2020;230:119636.

22. Fu X, Yang Z, Deng T, Chen J, Wen Y, Fu X, Zhou L, Zhu Z, Yu C. A natural polysaccharide mediated MOF-based Ce6 delivery system with improved biological properties for photodynamic therapy. J Mater Chem B. 2020;8(7):1481-8.

23. Pan $X$, Wang $H$, Wang S, Sun $X$, Wang L, Wang W, Shen H, Liu H. Sonodynamic therapy (SDT): a novel strategy for cancer nanotheranostics. Sci China Life Sci. 2018;61(4):415-26.

24. Son S, Kim JH, Wang X, Zhang C, Yoon SA, Shin J, Sharma A, Lee MH, Cheng L, Wu J, et al. Multifunctional sonosensitizers in sonodynamic cancer therapy. Chem Soc Rev. 2020;49(11):324461.

25. Gushchina OI, Larkina EA, Nikolskaya TA, Mironov AF. Synthesis of amide derivatives of chlorin e6 and investigation of their biological activity. J Photochem Photobiol B. 2015;153:76-81.

26. An J, Hu YG, Cheng K, Li C, Hou XL, Wang GL, Zhang XS, Liu B, Zhao YD, Zhang MZ. ROS-augmented and tumor-microenvironment responsive biodegradable nanoplatform for enhancing chemosonodynamic therapy. Biomaterials. 2020;234:119761.

27. Zhou Z, Song J, Nie L, Chen X. Reactive oxygen species generating systems meeting challenges of photodynamic cancer therapy. Chem Soc Rev. 2016;45(23):6597-626.

28. Yu J, Li S, Qi J, Chen Z, Wu Y, Guo J, Wang K, Sun X, Zheng J. Cleavage of GSDME by caspase-3 determines lobaplatin-induced pyroptosis in colon cancer cells. Cell Death Dis. 2019;10(3):193. 
29. Bertrand N, Wu J, Xu X, Kamaly N, Farokhzad OC. Cancer nanotechnology: the impact of passive and active targeting in the era of modern cancer biology. Adv Drug Deliv Rev. 2014;66:2-25.

30. Sun T, Zhang YS, Pang B, Hyun DC, Yang M, Xia Y. Engineered nanoparticles for drug delivery in cancer therapy. Angew Chem Int Ed Engl. 2014;53(46):12320-64.

31. Xu H, Yang D, Cai C, Gou J, Zhang Y, Wang L, Zhong H, Tang X. Dual-responsive mPEG-PLGA-PGlu hybrid-core nanoparticles with a high drug loading to reverse the multidrug resistance of breast cancer: an in vitro and in vivo evaluation. Acta Biomater. 2015;16:156-68.

32. Harrison H, Pegg HJ, Thompson J, Bates C, Shore P. HIF1-alpha expressing cells induce a hypoxiclike response in neighbouring cancer cells. BMC Cancer. 2018;18(1):674.

33. Robertson ED, Semenchenko K, Wasylyk B. Crosstalk between Mdm2, p53 and HIF1-alpha: distinct responses to oxygen stress and implications for tumour hypoxia. Subcell Biochem. 2014;85:199214.

34. Masoud GN, Li W. HIF-1alpha pathway: role, regulation and intervention for cancer therapy. Acta Pharm Sin B. 2015;5(5):378-89.

35. Ke Q, Costa M. Hypoxia-inducible factor-1 (HIF-1). Mol Pharmacol. 2006;70(5):1469-80.

36. Wang Y, Shi P, Chen Q, Huang Z, Zou D, Zhang J, Gao X, Lin Z. Mitochondrial RoS promote macrophage pyroptosis by inducing GSDMD oxidation. J Mol Cell Biol. 2019;11(12):1069-82.

37. Zhang Y, Su SS, Zhao S, Yang Z, Zhong CQ, Chen X, Cai Q, Yang ZH, Huang D, Wu R, et al. RIP1 autophosphorylation is promoted by mitochondrial ROS and is essential for RIP3 recruitment into necrosome. Nat Commun. 2017;8:14329.

38. Teng J-F, Mei Q-B, Zhou X-G, Tang Y, Xiong R, Qiu W-Q, Pan R, Law BY-K, Wong VK-W, Yu C-L, et al: Polyphyllin VI Induces Caspase-1-Mediated Pyroptosis via the Induction of ROS/NF-
KB/NLRP3/GSDMD Signal Axis in Non-Small Cell Lung Cancer. Cancers 2020, 12(1).

39. Fang Y, Tian S, Pan Y, Li W, Wang Q, Tang Y, Yu T, Wu X, Shi Y, Ma P, et al. Pyroptosis: A new frontier in cancer. Biomed Pharmacother. 2020;121:109595.

40. Jorgensen I, Miao EA. Pyroptotic cell death defends against intracellular pathogens. Immunol Rev. 2015;265(1):13.

41. Tang YS, Zhao YH, Zhong Y, Li XZ, Pu JX, Luo YC, Zhou QL. Neferine inhibits LPS-ATP-induced endothelial cell pyroptosis via regulation of ROS/NLRP3/Caspase-1 signaling pathway. Inflamm Res. 2019;68(9):727-38.

42. Dong W, Zhu Q, Yang B, Qin Q, Wang Y, Xia X, Zhu X, Liu Z, Song E, Song Y. Polychlorinated Biphenyl Quinone Induces Caspase 1-Mediated Pyroptosis through Induction of Pro-inflammatory HMGB1TLR4-NLRP3-GSDMD Signal Axis. Chem Res Toxicol. 2019;32(6):1051-7.

43. Cheng YC, Chu LW, Chen JY, Hsieh SL, Chang YC, Dai ZK, Wu BN. Loganin Attenuates High GlucoseInduced Schwann Cells Pyroptosis by Inhibiting ROS Generation and NLRP3 Inflammasome Activation. Cells 2020, 9(9).

44. Schroder K, Tschopp J. The inflammasomes. Cell. 2010;140(6):821-32. 
45. Zhou R, Yazdi AS, Menu P, Tschopp J. A role for mitochondria in NLRP3 inflammasome activation. Nature. 2011;469(7329):221-5.

46. Wu X, Zhang H, Qi W, Zhang Y, Li J, Li Z, Lin Y, Bai X, Liu X, Chen X, et al. Nicotine promotes atherosclerosis via ROS-NLRP3-mediated endothelial cell pyroptosis. Cell Death Dis. 2018;9(2):171.

47. Herr DR, Yam TYA, Tan WSD, Koh SS, Wong WSF, Ong WY, Chayaburakul K. Ultrastructural Characteristics of DHA-Induced Pyroptosis. Neuromolecular Med. 2020;22(2):293-303.

\section{Figures}
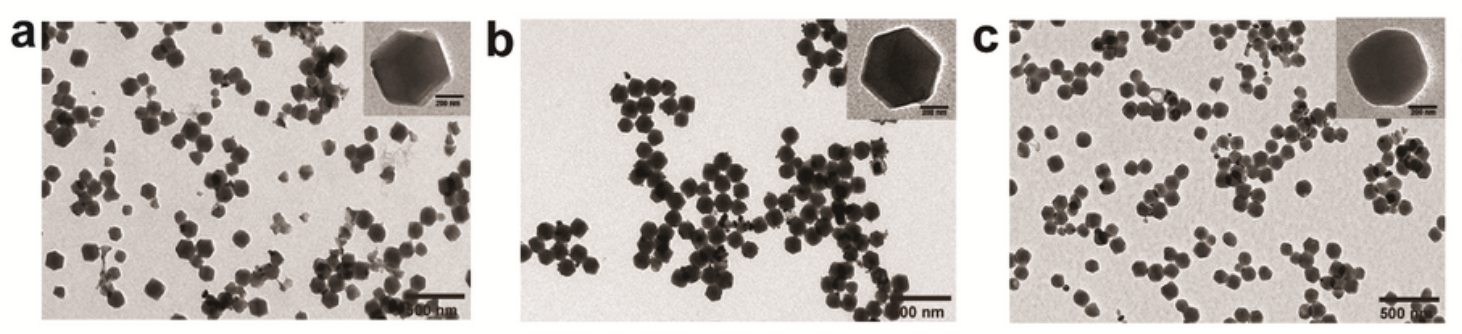

\section{g}
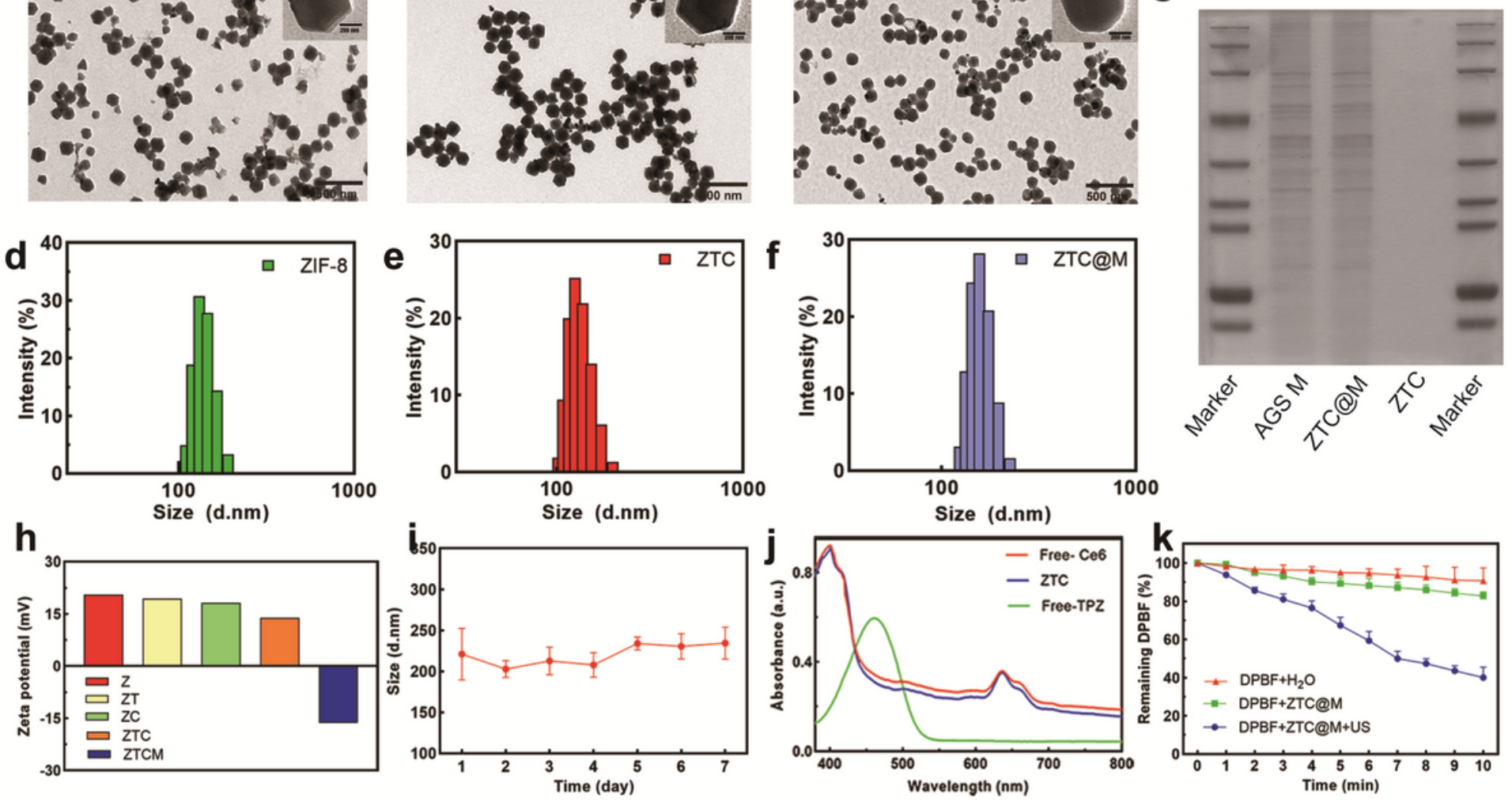

Figure 1

Characterization of ZTC@M nanoparticles. TEM and magnification images of ZIF-8 (a), ZTC (b) and ZTC@M (c). Scale bar is $500 \mathrm{~nm}$. Hydrated nanoparticle size distribution of ZIF-8 (d), ZTC (e) and ZTC@M (f).Gel Electrophoresis of AGS cytomembrane and ZTC@M (g). Zeta potential of different nanoparticles (h). The change of hydrated nanoparticle size of ZTC@M (i). The ultraviolet-visible absorption spectra of Ce6, TPZ and ZTC (j). Reactive oxygen species (ROS) production by ZTC under different conditions $(k)$. 

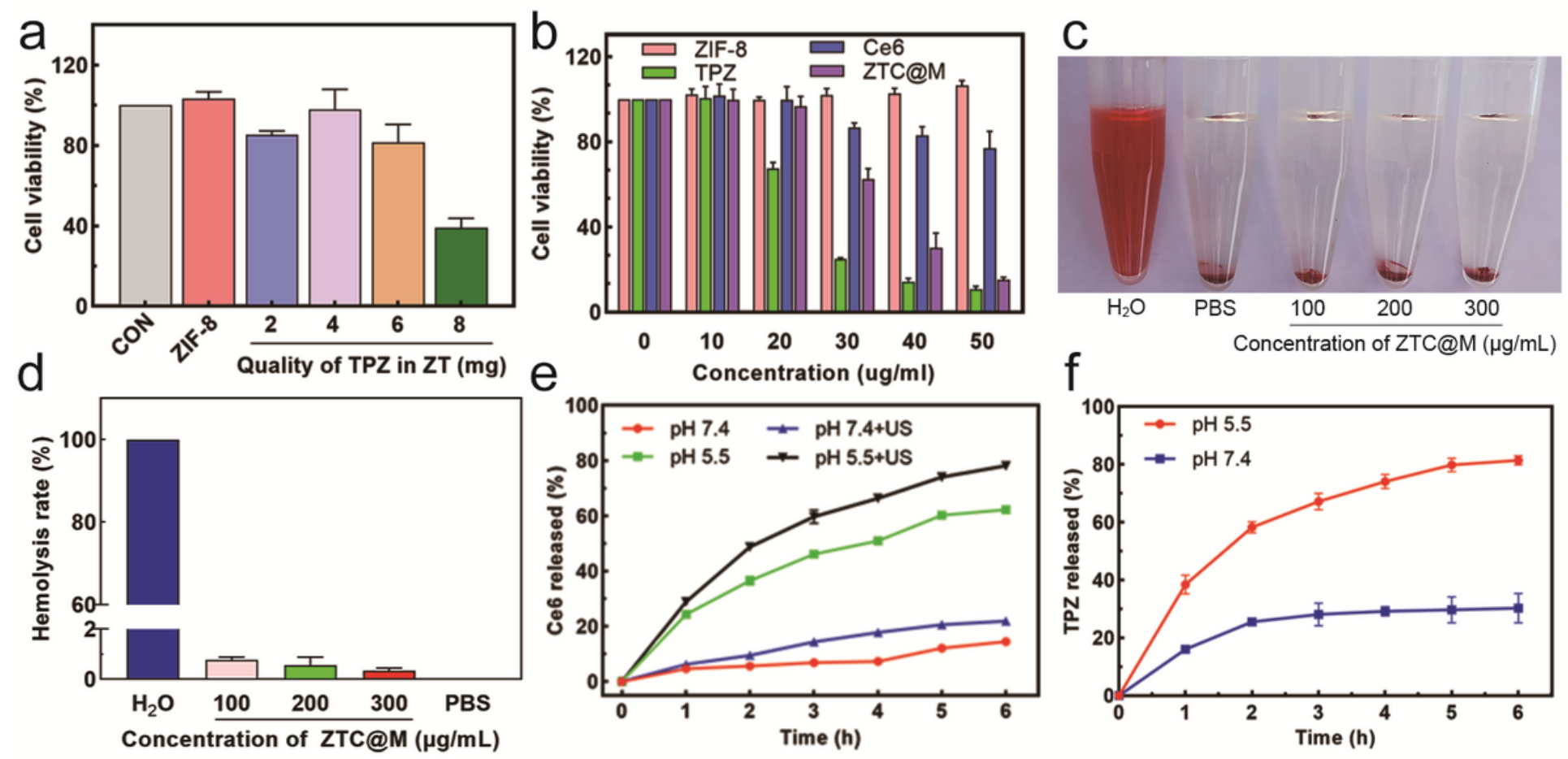

Figure 2

Cell viabilities of AGS cells incubated with ZTC at different loading concentration of TPZ (a). Cell viabilities of AGS cells treated with various probes (b). Hemolysis Assay of ZTC@M at different concentration (c) and hemolysis ratio of red blood (d). Ce6 controlled release curves of ZTC@M triggered by different $\mathrm{pH}$ and with/without US (e). 


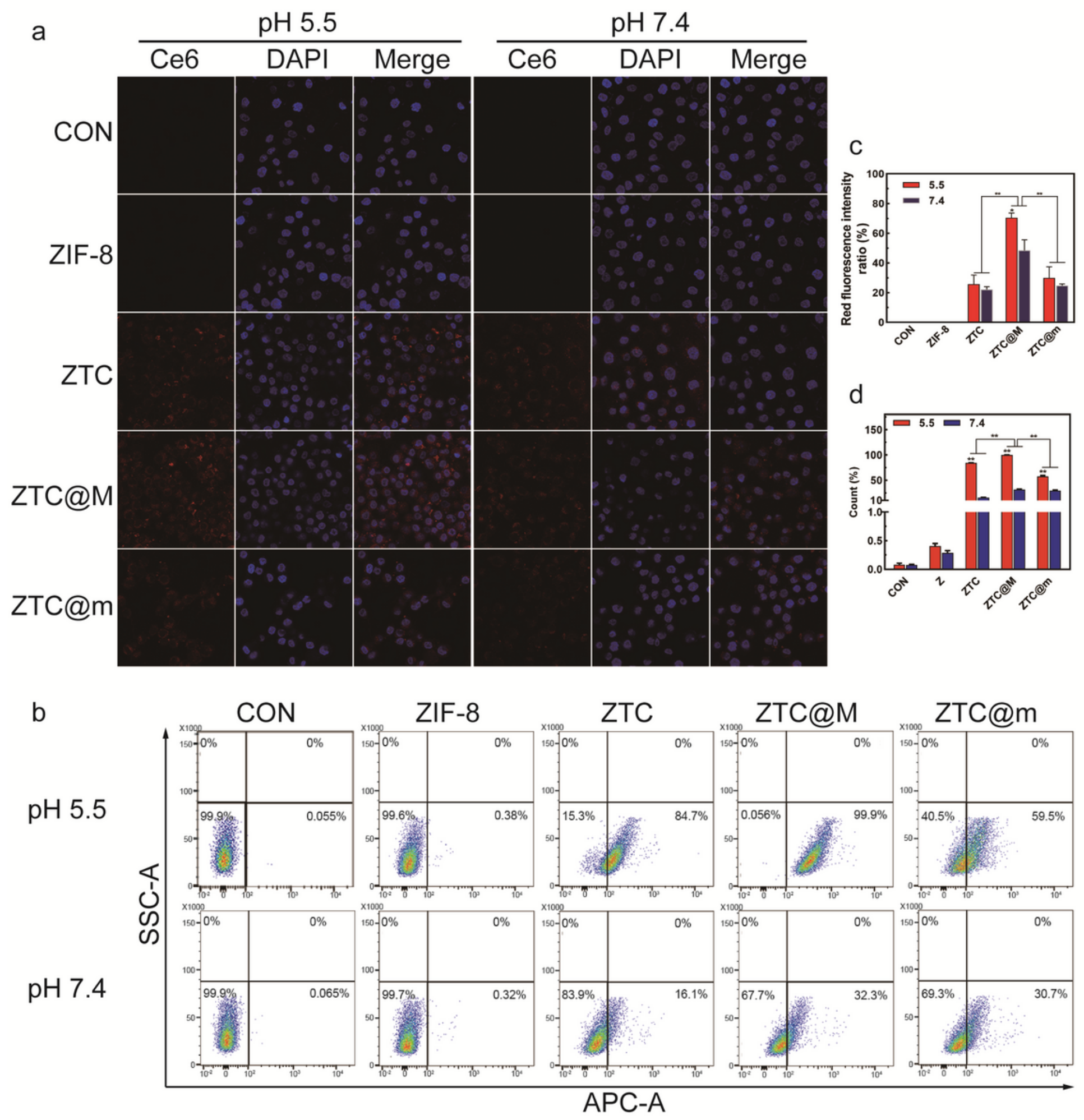

Figure 3

Cellular uptake experiments. Fluorescence imaging (a) and flow cytometry (quantitative detection of intracellular Ce6 fluorescence) (b) of AGS cells incubated with different nanoparticles at pH 7.4 and 5.5, respectively (600x). Quantification of intracellular fluorescence intensity in a (c). Count of AGS cells with intracellular fluorescence of $b(d) . *$ : $p<0.05, * * p<0.0001$. 
a

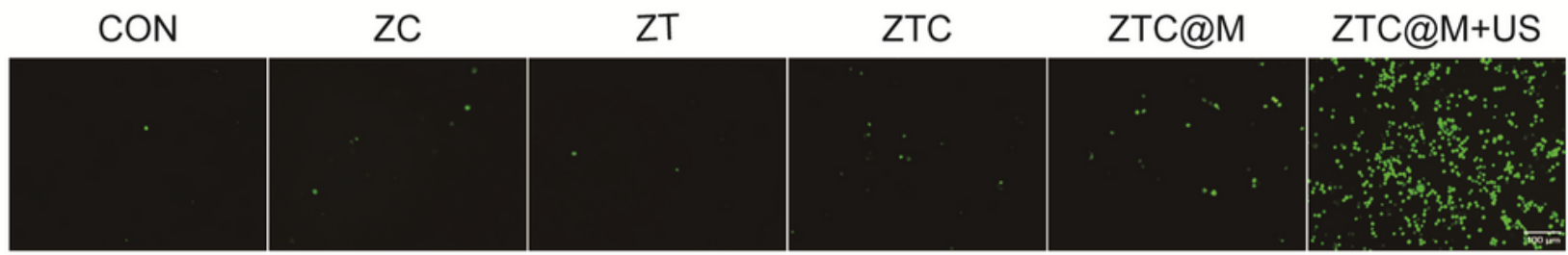

b

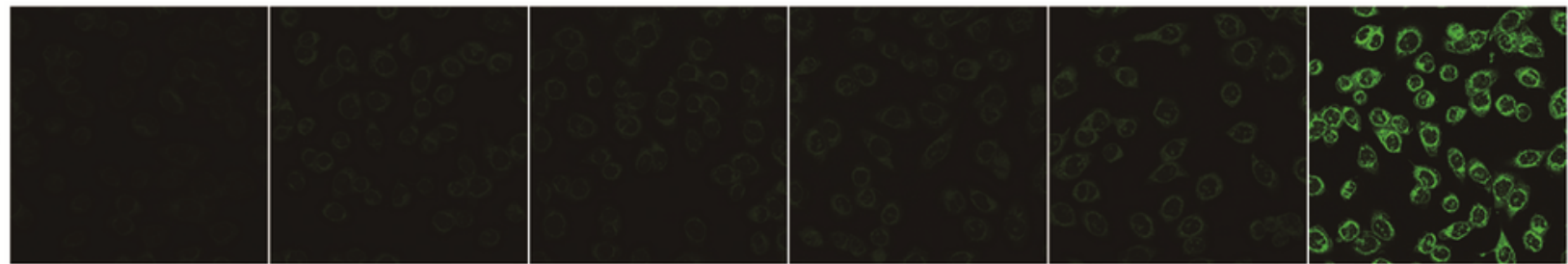

Merge

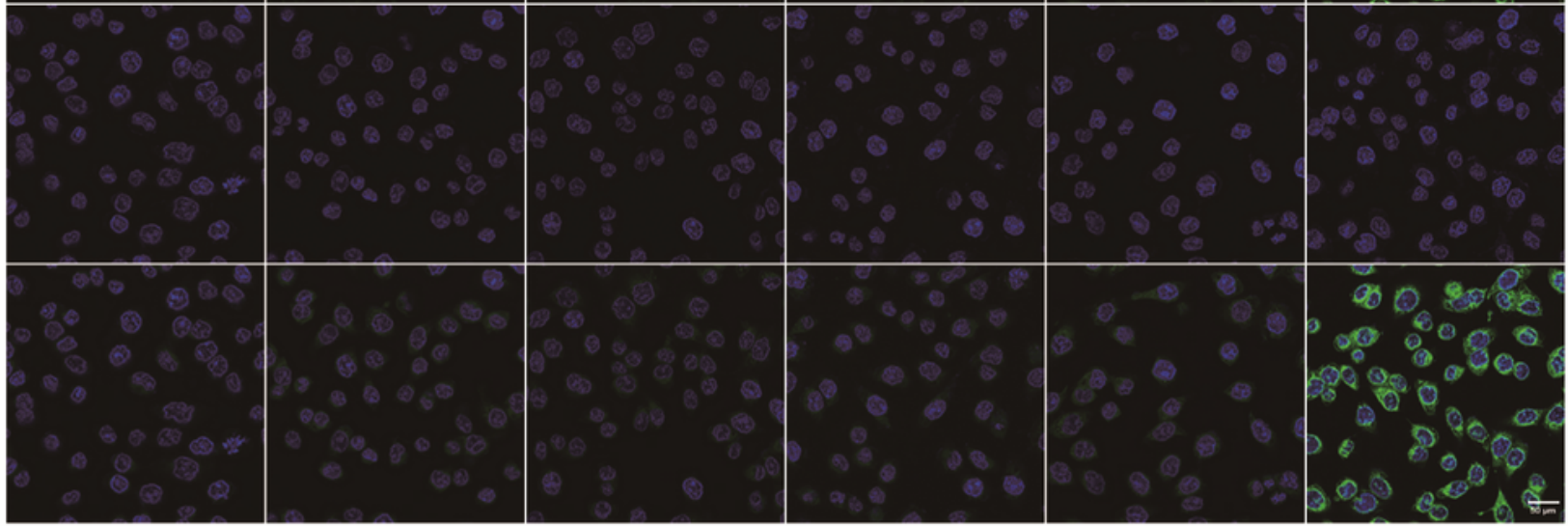

C

HIF-1a

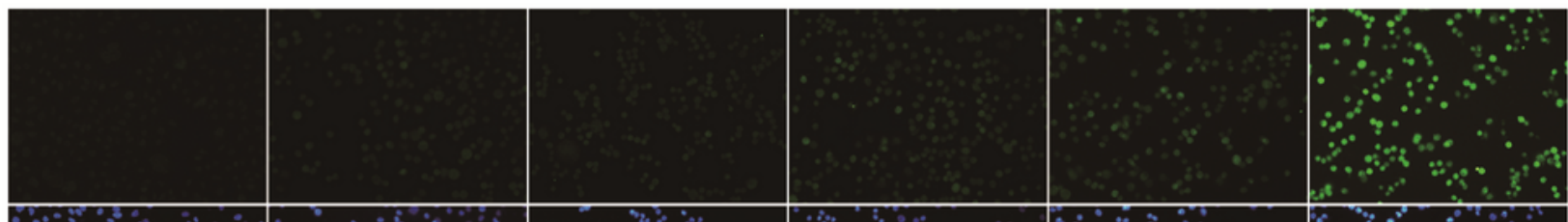

DAPI

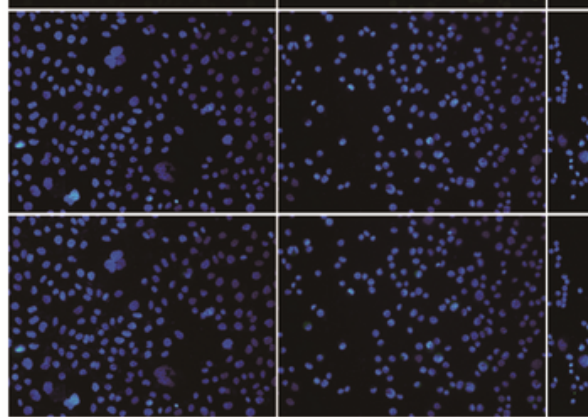

d

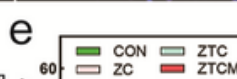

HIF-1a

GAPDH

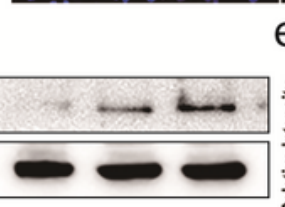

${ }_{60}=\mathrm{CON}_{\mathrm{ZC}}^{\mathrm{ZTC}}$
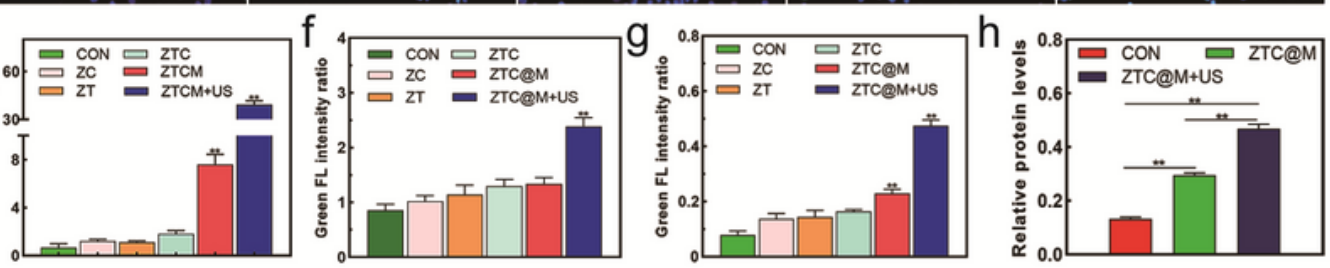

Figure 4

In vitro ROS/ hypoxia assay and IF. ROS production detected by fluorescence of DCFH-DA in AGS cells (a) (100x). Intracellular hypoxia imaging using Image-iт ${ }^{\text {тм }}$ Green Hypoxia Reagent as indicator (b) (200x). Immunofluorescence images of AGS stained by HIF-1a (c) (200x). Gel images of HIF-1a in AGS cells treated with ZTC@M+US (d). Quantification of intracellular fluorescence intensity in a (e), b (f) and c (g). Bar chart indicates the relative density of HIF-1 a to GAPDH in d (h). ${ }^{\star} p<0.05,{ }^{*} p<0.0001$. 
a
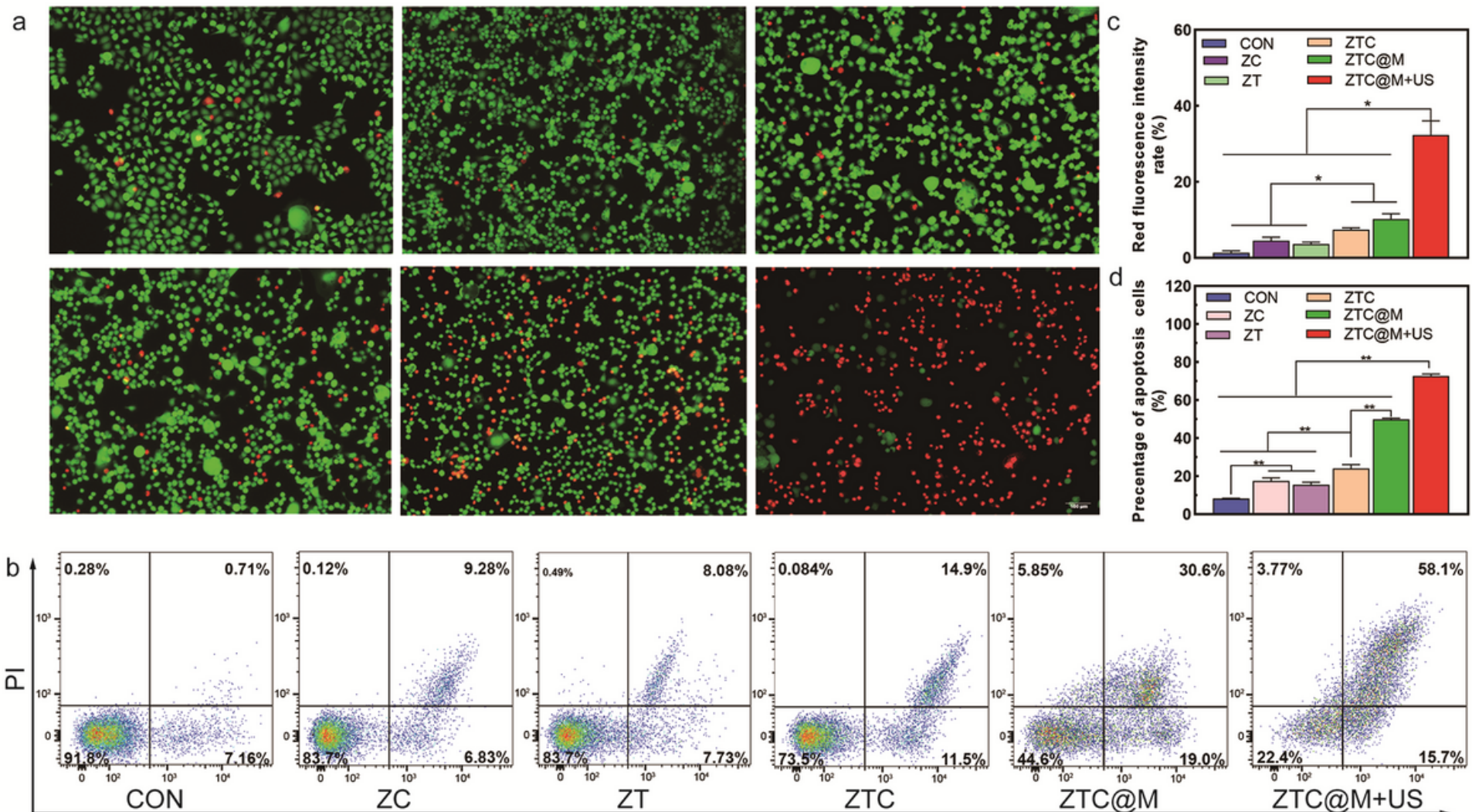

ZTC

Annexin-V FITC

Figure 5

In vitro therapeutic effect. Calcein-AM and PI stainned fluorescence images of AGS cells after various treatments (a) (100x). Flow cytometry analysis for apoptosis of AGS cells after various treatments (b). Quantification of intracellular fluorescence intensity in a (c). Bar chart indicates the percentage of apoptosis cells in $b(d) .{ }^{*} p<0.05, * * p<0.0001$. 

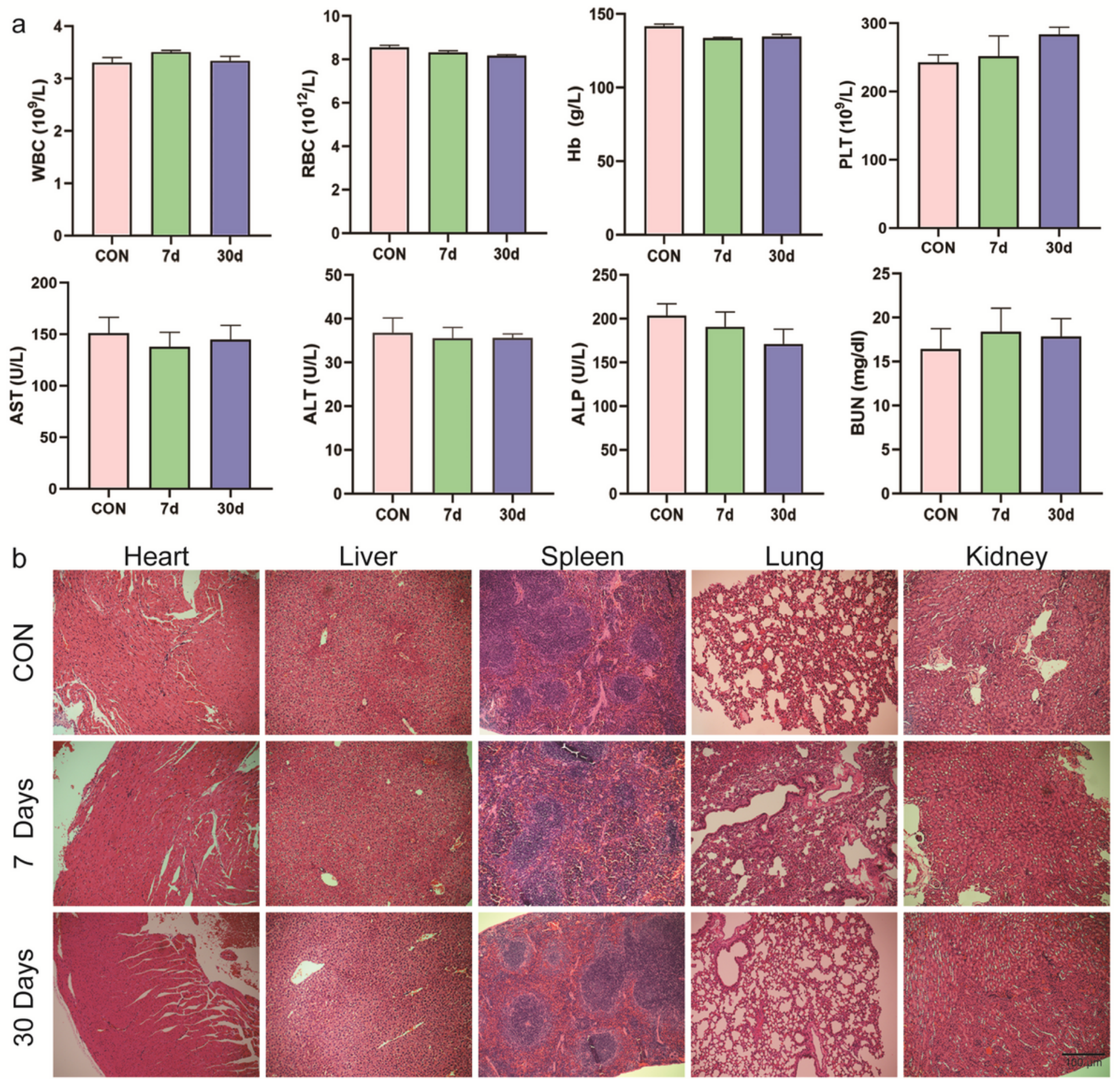

Figure 6

In vivo biocompatibility research. The count changes of WBC, RBC, Hb, PLT, ALT, AST, ALP and BUN (a). H\&E staining of nude mice after 7 and $30 \mathrm{~d}$ of three injections of ZTC@M (b) (100x). 
a

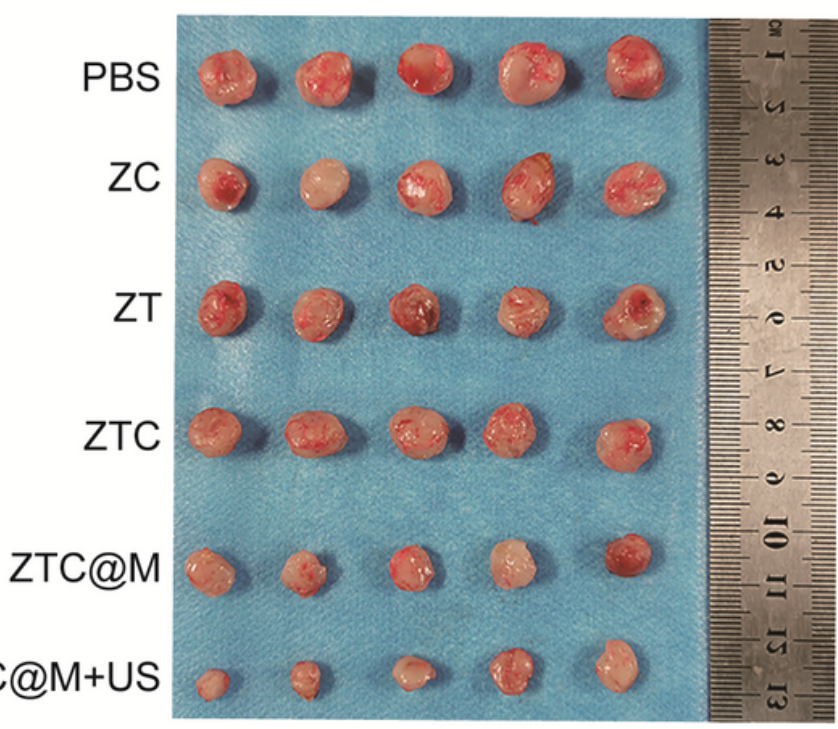

C

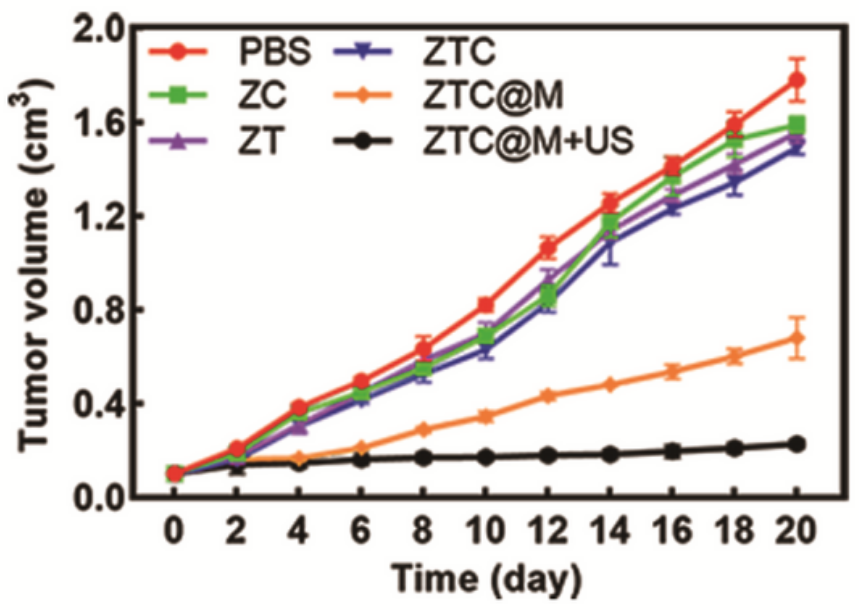

b
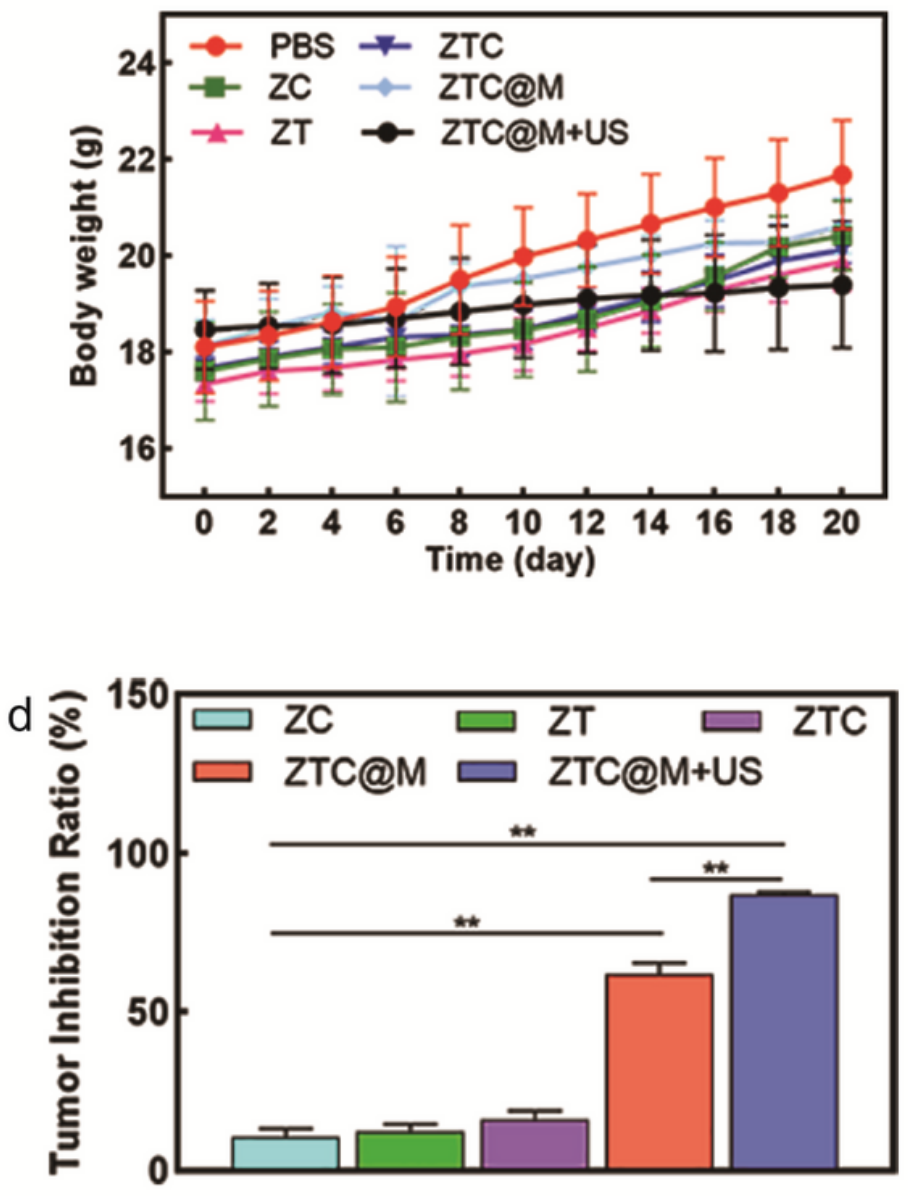
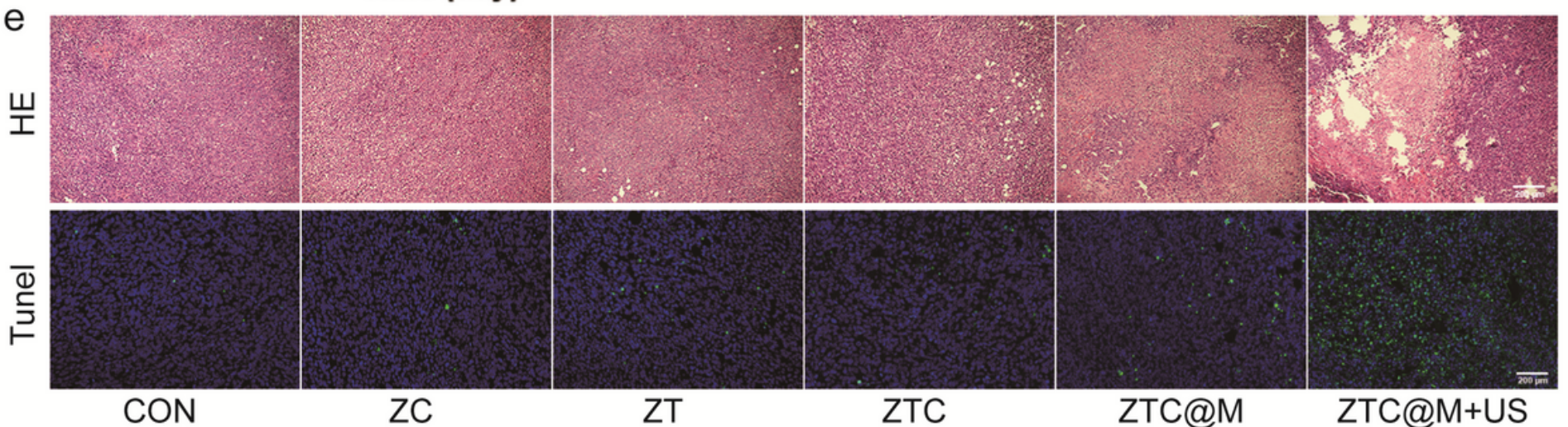

Figure 7

In vivo SDT therapy. Photos of tumor (a), tumor volume change (b), body weight change (c) and tumor inhibition rate (d) of AGS tumor-bearing nude mice with different treatments. H\&E and TUNEL staining of tumor after different treatments (e) (100x). $n=5 . *$ : $p<0.05, * *: p<0.0001$. 

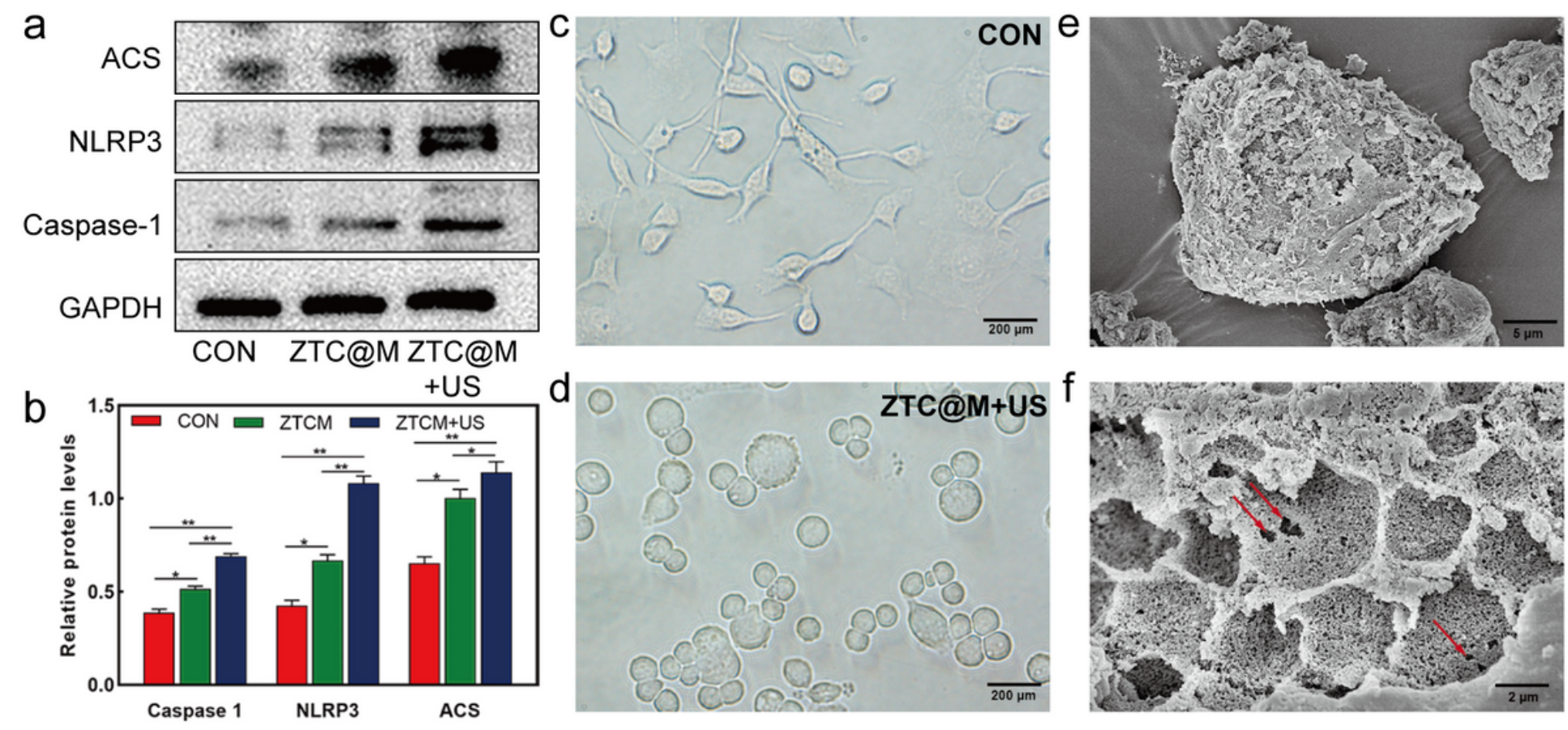

\section{Figure 8}

ZTC@M+US activates morphological and molecular characteristics of pyroptosis. The protein levels of caspase-1, ACS and NLRP3 after SDT (a). Bar chart indicates the relative density of caspase-1, ACS and NLRP3 to GAPDH in a (b). Phase-contrast photomicrographs showing AGS cells untreated (c) and treated with ZTC@M+US (d) (100x). Scanning electron micrographs of AGS cell after treatment with ZTC@M+US (e) and (f). Scale bars are $5 \mu \mathrm{m}(\mathrm{e})$ and $2 \mu \mathrm{m}(\mathrm{f}) .{ }^{\star} \mathrm{p}<0.05$, ${ }^{\star *} \mathrm{p}<0.0001$.

\section{Supplementary Files}

This is a list of supplementary files associated with this preprint. Click to download.

- Graphicalabstract.docx

- imageforthegraphicalabstract.tif 